\title{
Oculomotor Instabilities in Zebrafish Mutant belladonna: A Behavioral Model for Congenital Nystagmus Caused by Axonal Misrouting
}

\author{
Ying-Yu Huang, ${ }^{1,2}$ Oliver Rinner, ${ }^{2}$ Patrik Hedinger, ${ }^{1}$ Shih-Chii Liu, ${ }^{3}$ and Stephan C. F. Neuhauss ${ }^{1,2}$ \\ ${ }^{1}$ Institute of Zoology, ${ }^{2}$ Swiss Federal Institute of Technology (ETH) Zurich, Department of Biology, and Brain Research Institute, and ${ }^{3}$ Institute of \\ Neuroinformatics and ETH Zurich, University of Zurich, CH-8057 Zurich, Switzerland
}

\begin{abstract}
A large fraction of homozygous zebrafish mutant belladonna (bel) larvae display a reversed optokinetic response (OKR) that correlates with failure of the retinal ganglion cells to cross the midline and form the optic chiasm. Some of these achiasmatic mutants display strong spontaneous eye oscillations (SOs) in the absence of motion in the surround. The presentation of a stationary grating was necessary and sufficient to evoke SO. Both OKR reversal and SO depend on vision and are contrast sensitive.

We built a quantitative model derived from bel fwd (forward) eye behaviors. To mimic the achiasmatic condition, we reversed the sign of the retinal slip velocity in the model, thereby successfully reproducing both reversed OKR and SO. On the basis of the OKR data, and with the support of the quantitative model, we hypothesize that the reversed OKR and the SO can be completely attributed to RGC misrouting. The strong resemblance between the SO and congenital nystagmus (CN) seen in humans with defective retinotectal projections implies that $\mathrm{CN}$, of so far unknown etiology, may be directly caused by a projection defect.
\end{abstract}

Key words: zebrafish; reversed optokinetic response; congenital nystagmus; oculomotor instability; achiasmatic; retinal ganglion cell axons; axonal misrouting; quantitative model; behavioral genetics

\section{Introduction}

Zebrafish mutant belladonna (bel) was originally isolated in a screen of mutations affecting retinotectal axon pathfinding. It is named for an apparent dilated pupil caused by the retinal pigment epithelium (RPE) frequently not adjoining the lens (Karlstrom et al., 1996) (see Fig. 1). In a subsequent visual behavioral screen using optokinetic response (OKR), bel mutant larvae often displayed a sign-reversed OKR (i.e., the eyes moved opposite to the optical stimulus) (Neuhauss et al., 1999). Succeeding anatomical analysis of the retinotectal projection by injecting lipophilic tracer dyes revealed that all larvae with reversed OKR were achiasmatic (Rick et al., 2000). The reliable concurrence of these two phenotypes implies a strong link between the achiasmatic condition and the reversed OKR.

Recently, bel was found to be caused by a mutation in the zebrafish Lhx2 homolog, a Lim domain homeobox transcription factor required for forebrain patterning and midline axon guidance (Seth et al., 2006). In the mid-1990s, an achiasmatic condition was first reported in humans (Apkarian et al., 1994). This rare heritable disease, termed nondecussating retinal-fugal fiber

Received March 16, 2006; accepted Aug. 4, 2006.

This work was supported by the Swiss National Science Foundation (S.C.F.N.), the Velux Foundation (S.C.F.N.), and the European Molecular Biology Organization Young Investigator Program (S.C.F.N.). S.C.F.N. was a Schweizerischer Nationalfonds Förderungsprofessor. We would like to thank Drs. Martin Schwab and Jean-Marc Fritschy for discussions. We also thank David Belet and Hansjörg Kasper for technical assistance.

Correspondence should be addressed to Stephan C. F. Neuhauss, Institute of Zoology, University of Zurich, Winterthurerstrasse 190, CH-8057 Zurich, Switzerland. E-mail: stephan.neuhauss@zool.unizh.ch.

DOI:10.1523/JNEUROSCI.2886-06.2006

Copyright $\odot 2006$ Society for Neuroscience $\quad$ 0270-6474/06/269873-08\$15.00/0
(NDRFF) syndrome, is frequently accompanied by oculomotor instabilities, with horizontal and see-saw nystagmus (Apkarian et al., 1995; Korff et al., 2003). The OKR profile in achiasmatic patients looked rather complex and appears not to be reversed (Apkarian and Bour, 2001). In addition to the reported cases in humans, an achiasmatic strain of Black Belgian sheepdogs was identified (Williams et al., 1994; Hogan and Williams, 1995). Analogous to achiasmatic humans, the absence of the optic chiasm in this canine led to congenital nystagmus (CN) (Dell'Osso and Williams, 1995; Dell'Osso et al., 1998).

Reversed OKR, on the other hand, has been described in albino humans (St John et al., 1984; Collewijn et al., 1985) and albino rabbits (Collewijn and Grootendorst, 1978). Interestingly, albinism is a condition that has been associated with altered patterns of retinal ganglion cell (RGC) decussation in the optic chiasm and deficits in eye morphogenesis (Jeffery, 1997). Moreover, reversed OKR has also been observed in normally pigmented humans where it mostly co-occurs with CN (Halmagyi et al., 1980). However, the underlying optic projection was not analyzed in these patients.

In human studies, visual dysfunctions caused by the achiasmatic defect were merely based on case reports. With the exception of the canine strain, investigators had to depend on surgically induced ipsilateral projections of RGC axons to study the achiasmatic condition in animals (Easter and Schmidt, 1977). bel provides a robust model for repeated measurements of eye movements related to the influence of the RGC misrouting. The zebrafish is a monocular vertebrate with RGCs completely pro- 
jecting to the contralateral, or in the case of achiasmatic bel, to the ipsilateral side of the brain. Thus, in contrast to binocular primates and most mammals, the effects of the RGC misrouting can be studied without the complication of a mixed projection.

In this study, we report a novel feature in achiasmatic bel larvae: spontaneous eye oscillations ( $\mathrm{SOs}$ ) that closely resemble $\mathrm{CN}$ in human patients. We describe the oculomotor abnormalities observed in these achiasmatic bel mutants. Furthermore, we replicate the reversed and oscillatory eye movements in a simple quantitative model to confirm that the achiasmatic condition is sufficient to explain these abnormal eye behaviors.

\section{Materials and Methods}

Fish maintenance and breeding. The bel (bel $\left.{ }^{\text {tv42 }}\right)$ mutant line was maintained and bred as described previously (Mullins et al., 1994). Outcrossed sibling pairs were set up to identify heterozygous carriers. Clutches of these identification crosses as well as crosses of already identified carriers were used to assess visual behavior. Embryos were raised at $28^{\circ} \mathrm{C}$ in E3 medium (in mM: $5 \mathrm{NaCl}, 0.17 \mathrm{KCl}, 0.33 \mathrm{CaCl}_{2}$, and $0.33 \mathrm{MgSO}_{4}$ ) (Haffter et al., 1996) and staged according to development in days postfertilization (dpf). Larvae at 5-7 dpf were anesthetized with 3-aminobenzoic acid ethyl esther methane sulfonate to sort the homozygous mutants according to their eye pigmentation phenotype.

Histology. For Richardson staining, larvae were fixed in $4 \%$ paraformaldehyde, dehydrated through a graded series of ethanol-water mixture washes, and incubated in Technovit 7100 (Heraeus Kulzer, Wehrheim, Germany). After polymerization at room temperature, microtome sections $(3 \mu \mathrm{m})$ were prepared and mounted on SuperFrost Plus slides (Menzel-Gläser, Braunschweig, Germany), air dried at $60^{\circ} \mathrm{C}$, stained with toluidine blue solution $(0.1 \%$ in aqua dest.), overlaid with Entellan (Merck, Darmstadt, Germany), and coverslipped. Slides were examined under the light microscope.

OKR. The optokinetic system is driven by the global pattern of visual stimulation from which it extracts the magnitude and direction of the velocity relative to the retina (often called retinal slip) to generate an eye movement (OKR) equal in speed and opposite in direction, stabilizing the visual world on the retina. The OKR is characterized by a slow phase, during which time the eyes compensate for movement of the visual world, followed by a fast phase (saccade), which moves the eyes back from the limits of their orbital rotation to a more central position. At the beginning of a slow phase, the eye accelerates until reaching a steady state, which is the best possible compensation velocity for a given retinal slip. This will be referred to as the slow-phase velocity (SPV), in which decelerating occurs before the initiation of the subsequent saccade.

The method of optokinetic stimulation was performed as described previously (Rinner et al., 2005). Briefly, to suppress whole-body displacement without constricting eye movement, larvae were imbedded dorsal up in the center of a 35-mm-diameter Petri dish containing 3\% methylcellulose. Using a digital light projector (Proxima 4200 and HP vp6111), moving and still vertical sine-wave gratings were projected onto a cylindrical diffusion screen at varying contrast, stimulus velocity, and spatial frequency. The dimensions of the projected image were $99^{\circ}$ horizontally and $118^{\circ}$ vertically, whereas the distance of the screen to the larva's eye was approximately $4.65 \mathrm{~cm}$. To correct for the curvature, the gratings were wrapped onto the cylindrical screen through a mapping function. The projector output was linearized by means of look-up takes.

Only the right eye of the larva was stimulated. During stimulation, eye movements were recorded by an infrared-sensitive CCD camera. Frames were processed simultaneously by a custom-developed software (LabView IMAQ, version 5.1; National Instruments, Austin, TX) that is capable of retrieving the angular position and velocity of the larva's eyes. If not indicated otherwise, only the data of the stimulated eye were analyzed with a sample rate of 12.5 samples/s.

The code for the analysis of the eye recording was written in the $\mathrm{R}$ statistical computing language ( $\mathrm{R}$ Foundation for Statistical Computing, Vienna, Austria). Before statistically analyzing eye movements for each condition, the eye position $\left(s_{e}\right)$ time series was smoothened using a Gaussian smoothing kernel with $n=21$ and bandwidth $h=2.533$. Eye velocity $\left(v_{e}\right)$, and eye acceleration $\left(a_{e}\right)$ were computed with a simple derivation algorithm $\left[a_{e}(t)=S R\left[v_{e}(t)-v_{e}(t-1)\right]\right.$, where $S R$ denotes the sample rate]. For the differentiation between the slow phase and the fast phase of the OKR, the eye movement time series $\left(s_{e}, v_{e}\right.$, and $\left.a_{e}\right)$ were split with every segment spanning from one acceleration peak to the next. Fast-phase segments of the OKR (i.e., saccadic eye movements) were identified using visually determined eye acceleration thresholds on the eye acceleration peaks $\left(18 \% \mathrm{~s}^{2}\right.$ when the eye moved parallel to the stimulus velocity, $10 \% \mathrm{~s}^{2}$ when the eye moved in the opposite direction). The other segments were considered slow phases with a steady state if they had a minimum length of $1.2 \mathrm{~s}$. The reason for having that minimum duration requirement for the slow phases is that we wanted to make sure to only use those slow phases in which the eye reaches a steady eye velocity state. The SPV for one condition was computed by taking the maximum eye velocity across all slow phases in a condition. For the statistical analysis of the OKR in general, all identified slow-phase segments of $s_{e}, v_{e}$, and $a_{e}$ in one condition were treated as one data row in which the descriptive statistics were calculated and then pulled together and grouped based on their OKR phenotype [wild type (wt), bel forward $(f w d)$, and bel reverse ( rev)] to calculate the respective statistics for each group.

Experimental procedure. If not noted otherwise, the following stimulation has been used. The spatial frequency of the sine-wave grating was 0.045 contrast/degree (c/deg). Contrast was varied on an interval between 0 and $99 \%$ as derived by the following: $c_{s}=\left(I_{\max }-I_{\min }\right) /\left(I_{\max }+\right.$ $\left.I_{\min }\right) 100$, where $I_{\max }$ denotes the maximum illumination $\left(5230 \mathrm{~cd} / \mathrm{m}^{2}\right)$ and $I_{\text {min }}$ denotes the minimum illumination in the sine-wave grating. The maximum contrast achieved by the projector will be referred to as $100 \%$ instead of $99 \%$ to improve readability. For measuring the OKR at different stimulus velocities, we used a back-and-forth moving sine-wave grating with the direction changing every $5 \mathrm{~s}$.

Quantitative model. We built a quantitative model of the OKR using MATLAB Simulink (Mathworks, Natick, MA) (see Fig. 6). The parameter estimation and details on the derivation of the model are presented in supplemental Tables 1 and 2, and the supplemental movie (available at www.jneurosci.org as supplemental material).

The model contains fast-phase (saccade) and slow-phase circuits that are alternately active. This corresponds to the experimentally derived data in which the slow phase of the OKR is frequently interrupted with saccades to reset the eye after approaching the maximum eye range. The slow phase of the OKR is modeled with the OKR and the eye velocitydependent load (Ve Load block). In wt, the OKR serves the purpose of stabilizing the visual image on the retina when the visual scene moves relative to the retina of the fish. Accordingly, in the OKR model, the retinal slip velocity is the error signal to minimize the retinal slip. The OKR block generates eye accelerations depending on the magnitude of the retinal slip velocity (see Fig. $6 C$ ). Eye acceleration is then integrated to obtain a new eye velocity, which in turn leads to a new and smaller retinal slip. This loop would eventually reduce the retinal slip to zero, which is in contrast to the experimental data in which a steady state is reached with a residual retinal slip (steady-state error), or in other words, with a slowphase gain (SPG) $<1$ (see Fig. 3B). Therefore, we modified the model by placing a Ve Load block on the loop to prevent the slow-phase circuit from perfectly minimizing the retinal slip.

The fast phase of the OKR (saccade) was modeled in the saccade system block. Whenever the eye surpasses a predefined eye position limit during the slow phase, the model switches to the fast-phase loop to reset the eye. After termination of the saccade, the switches are reset with the slow-phase loop taking over. Eye position and velocity are copied at the end of the fast phase by the slow-phase loop as both systems overlap between the two switches.

Statistical analysis. To test the main effect of stimulus contrast on the SPV, we performed a two-way repeated-measures (RM) ANOVA for each group (wt, mut fwd, and mut rev) with stimulus contrast and stimulus velocity as within-subject factors. We then used a mixed RMANOVA with group as the between-subject factor and stimulus velocity as the within-subject factor to test whether there was a significant difference in contrast sensitivity between groups. Fish with unrealistically high-contrast sensitivities of $>100$ were not included in the analysis. The 

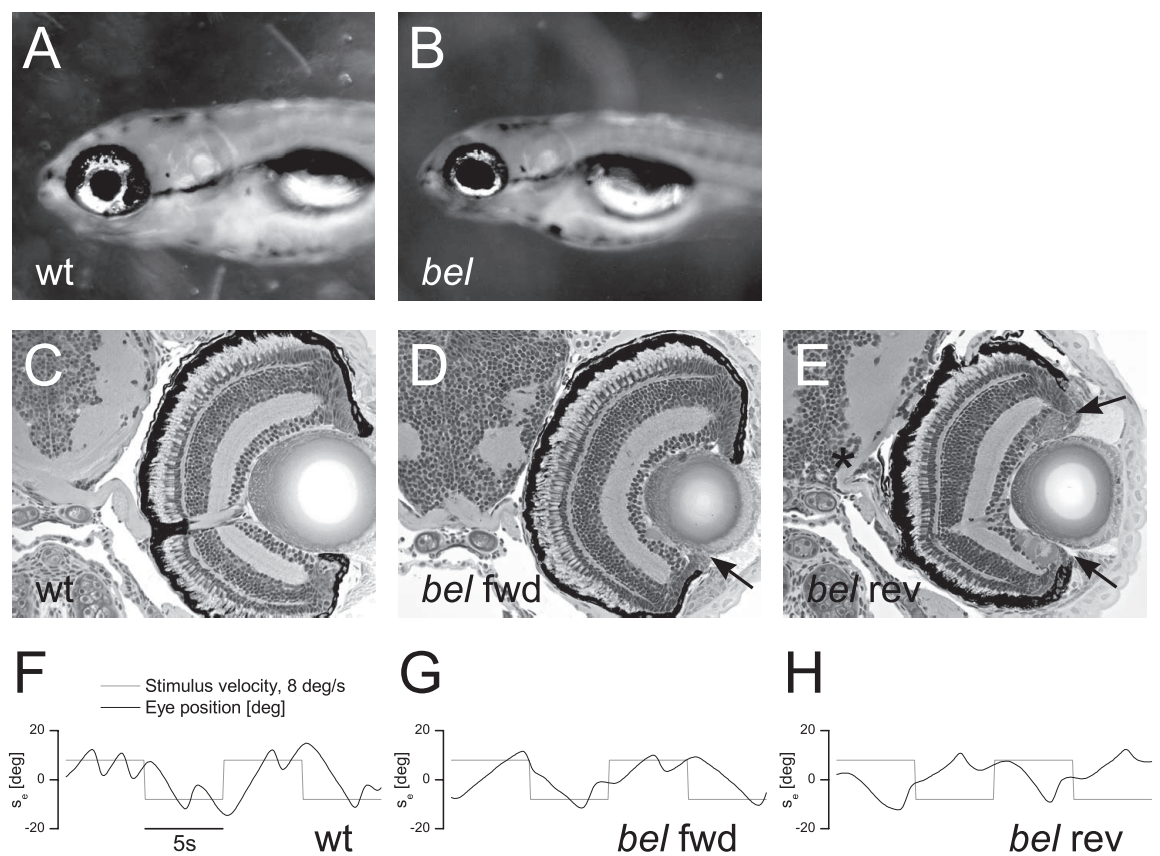

Figure 1. Phenotypes of bel larvae at $5 \mathrm{dpf} . \boldsymbol{A}, \boldsymbol{B}, \mathrm{A}$ gap between the lens and the pigmented epithelium of the eye gives the appearance of enlarged pupils in bel larvae $(\boldsymbol{B}) . \boldsymbol{C}-\boldsymbol{E}$, Transverse sections of the retina and brain. The ipsilateral projection of the optic nerve of bel rev is shown with the asterisk. Arrows indicate the pigmentation defects both in bel fwd and bel rev. $\mathbf{F}-\mathbf{H}, \mathbf{O K R}$ sample traces of eye position after stimulation with moving gratings $\left(8^{\circ} / \mathrm{s}\right)$.
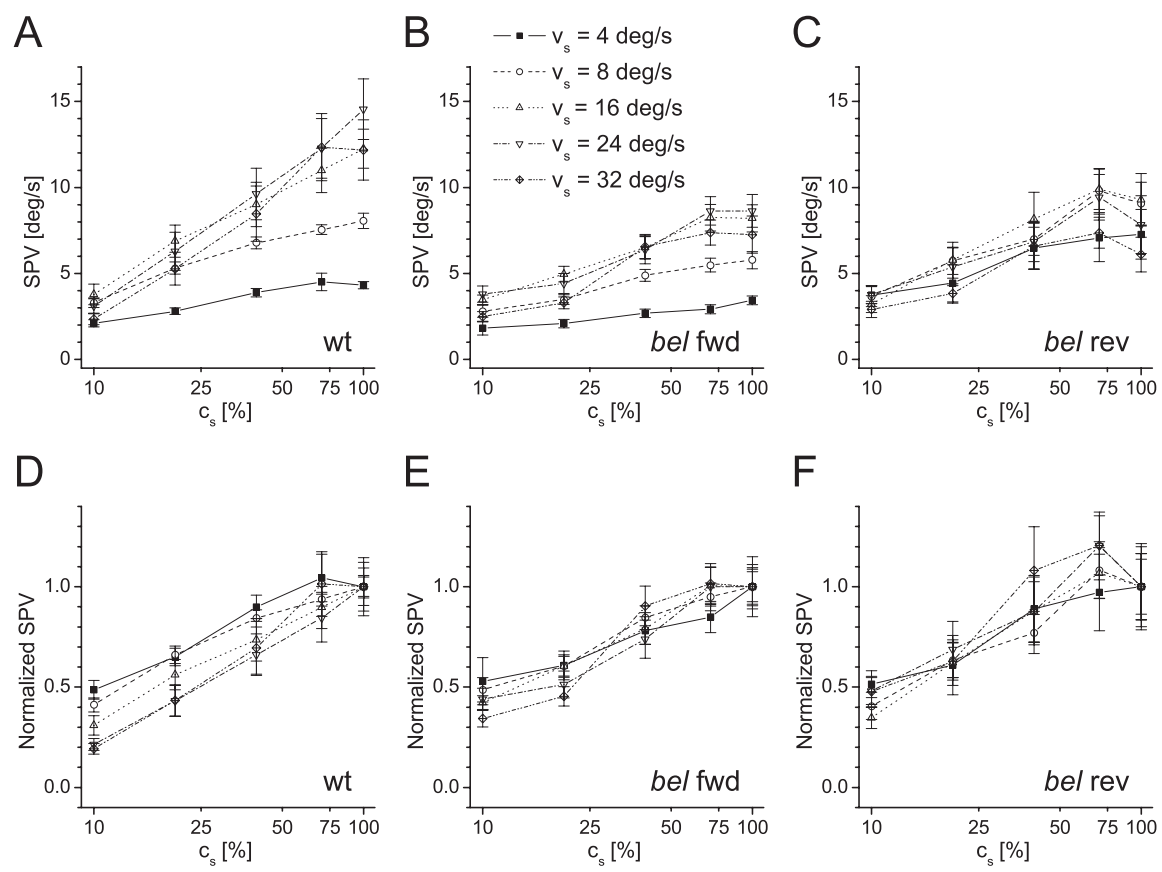

Figure 2. SPV as a function of logarithmic stimulus contrast $\left(c_{s}\right) . A-C$, A series of stimulus contrast $\left(\boldsymbol{C}_{s}=10,20,40,70\right.$, and $100 \%$ ) was applied to assess the influence of stimulus contrast on SPV. Contrast dependence of SPV was measured at varying stimulus velocity $\left(v_{s}=4,8,16\right.$, and $\left.24^{\circ} / s\right)$ of wt $(n=9 ; \boldsymbol{A})$, bel fwd $(n=9 ; \boldsymbol{B})$, and bel rev $(n=9 ; \boldsymbol{C})$. $\boldsymbol{D}-\boldsymbol{F}$, Same data displayed as normalized SPV in wt $(\boldsymbol{D})$, in bel fwd $(\boldsymbol{E})$, and in bel rev $(\boldsymbol{F})$. The normalized $S P V$ was calculated as $S P V\left(c_{s}\right) / S P V\left(c_{s}=100 \%\right)$. SPV among all groups showed a positive linear relationship to the logarithmic stimulus contrast.

same statistical method was used to test whether the three groups differed on saccade peak velocity. Because mixed RM-ANOVA cannot handle missing data, some fish had to be excluded from the analysis.

\section{Results}

The zebrafish mutant bel is a recessive mutation named after its "enlarged" pupils caused by an RPE defect (Fig. 1A,B) (Wilhelm et al., 1991; Karlstrom et al., 1996; Feinsod, 2000). This prominent feature is a consequence of the RPE failing to adjoin the lens, thereby leaving a visible gap (Fig. $1 \mathrm{D}, \mathrm{E}$, asterisk). Approximately $40 \%$ of $\mathrm{bel}$ display a reversed OKR (bel rev), which is when a clockwise moving stimulus elicits counterclockwise eye movements while the remaining larvae have a normal sign OKR ( bel fwd). This behavioral abnormality correlates perfectly with noncrossing optic fibers at the midline. Hence all bel rev are achiasmatic (Rick et al., 2000). Despite an equivalent pigmentation defect in bel rev and bel fwd, the optic nerves of the latter cross normally at the midline and form an optic chiasm. bel rev, in contrast, have an achiasmatic phenotype with the optic fibers projecting into the ipsilateral brain hemispheres (Fig. 1C-E, arrows). Figure $1 \mathrm{~F}-\mathrm{H}$ shows the OKR traces typically generated by the three phenotypes. Frequently interrupted through fast resets (saccades), the eyes of wt move in the same direction and approximately at the same velocity as the stimulus (Fig. $1 F$ ). The OKR geometry of bel fwd looks very similar to that of wt as the eyes of bel fwd move parallel to the stimulus during the slow phase, although the overall velocity and the number of saccades per time unit are reduced (Fig. 1G). Finally, the waveform of the reversed OKR is presented in Figure $1 \mathrm{H}$. Aside from the reduced overall velocity, the most characteristic feature of the OKR of bel rev is the antiparallel eye movement to the stimulus.

\section{Stimulus contrast $\left(c_{s}\right)$ sensitivity}

The OKR gain (eye velocity/stimulus velocity) linearly correlates with the logarithm of the stimulus pattern contrast regardless of spatial frequency in zebrafish larvae (Rinner et al., 2005). Because Rick et al. (2000) showed that the number of saccadic eye movements per time interval were unaffected by stimulus velocity changes in bel rev, it is conceivable that the eye movements are equally unaffected by $c_{s}$ manipulation. Such a result would be consistent with the notion that the reversed $\mathrm{OKR}$ is driven by a stimulus-independent internal pacemaker mechanism. Therefore, we investigated the effect of varying $c_{s}$ on the OKR of bel rev larvae. Figure $2 A-C$ shows that the SPV of bel rev $\left(F_{(4,32)}=\right.$ $18.513 ; p=0.000)$ exhibited a positive linear relationship with the logarithmic $c_{s}$ analogous to bel fwd $\left(F_{(4,32)}=36.737 ; p=0.000\right)$ and wt $\left(F_{(4,32)}=82.103 ; p=0.000\right)$. When plotting against the normalized SPV, it is even more evident that the gain of the OKR is affected much in the same way in all three types of fish (Fig. 2D-F). Then we calculated the threshold contrast by intersecting the regression line for each fish and 
group with the horizontal line at SPV = $1.5 \%$ (noise floor of the SPV data). The temporal contrast sensitivity (reciprocal of threshold contrast) (supplemental Table 2, available at www.jneurosci.org as supplemental material) is similar in all groups along different $\operatorname{SPV}\left(F_{(2,18)}=0.036 ; p=\right.$ $0.965)$. Hence, the reversed OKR is influenced in contrast and not independent on visual input.

\section{Stimulus velocity challenge}

Figure 2, $A$ and $B$, shows that, given the same contrast, both wt and bel fwd eye velocities raise with increasing stimulus velocity until they reach their peak at $32 \%$. These results extend the previous finding of Rick et al. (2000), who reported independence of gain and stimulus velocity. Our results confirm this abnormal relationship between input velocity and eye velocity. However, with more precise measurements, we observed a slight dependence on stimulus velocity in bel rev at low velocities of $4-16 \%$. The same effect is still present at reduced contrast (Fig. 2C). To verify this observation, we tested the OKR on an extensive range of stimulus velocities. Figure $3 A$ shows that the SPV of both wt and bel fwd correlated positively with low stimulus velocities (i.e., $<32 \%$ ), decreasing steadily thereafter. In bel rev, however, the SPV started at a much higher value and reached its maximum two steps earlier than wt and bel fwd, and it already began to decline at a stimulus velocity of $24^{\circ} \%$. The peak SPV was comparably lower in bel rev and bel fwd. Thus, the OKR of bel rev had significantly reduced stimulus velocity dependence with the SPG exceeding one when the stimulus velocity was $<16 \%$ s (Fig. $3 B$ ). This is in contrast to wt and bel fwd that only displayed gain values $\leq 1$ (Fig. $3 B)$.

We also compared the saccade peak velocity among the three groups to exclude the possibility that the optokinetic profile seen in bel rev and bel fwd is a result of a motor deficiency. The peak velocity reached during the saccades was similar in all three groups across stimulus velocities (Fig. $3 C$ ). Hence, the saccade system and the motor performance appears to be unaffected by the bel mutation. Additionally, to understand the cross-feed between the two brain hemispheres, we computed the velocity of the unstimulated eye as the fold change of the stimulated eye velocity. This value was, on average, 1.27 in bel rev, whereas in wt and bel fwd, it was 0.76 and 0.82 , respectively. The higher amplitude of the unstimulated eye in bel rev indicates that the major signal was fed into the ipsilateral hemisphere.

\section{SOs}

At the cessation of the moving grating, we made the intriguing observation of SOs in 20-80\% (different for all clutches) of bel rev (supplemental movie, available at www.jneurosci.org as supplemental material). Those fish exhibiting the SOs do so for most of the time as soon as they are able to perceive the pattern (ShalloHoffmann et al., 1999). As presented in Figure 4A, in addition to sporadic saccades, the eyes of wt stopped moving with the cessation of motion in the surround. Yet, the eyes of bel rev continued to be moving, displaying alternating waveforms of very different character compared with the reversed OKR (Fig. $4 B$ ). To investigate the properties of the $\mathrm{SO}$, we tested bel rev at different stimulus conditions. In contrast to wt, the eyes of bel rev keep oscil-
B

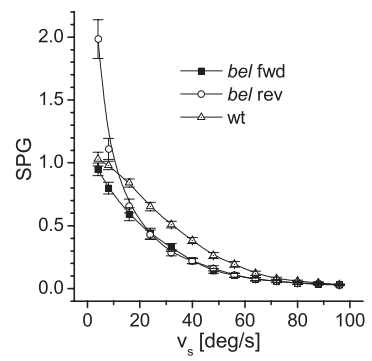

C

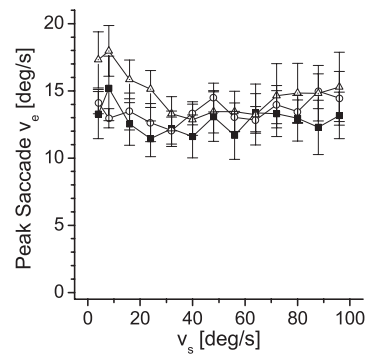

Figure 3. Stimulus velocity challenge of the OKR. $A$, The course of the SPV versus the stimulus velocity $\left.\left(v_{s}\right)=4 \%-96^{\circ} / \mathrm{s}\right)$ for wt $\left(n_{w t}=18\right)$, bel fwd $\left(n_{b f}=18\right)$, and bel rev $\left(n_{b r}=18\right)$ at a stimulus contrast of $c_{s}=100 \%$. B. Same data plotted as slow-phase gain $\left(S P G=S P V / v_{s}\right)$. The spatial frequency of the stimulus was $0.045 \mathrm{c} / \mathrm{deg}$, and the stimulus velocity direction alternated every did not sample sizes: $\left.n_{w t}=10, n_{b f}=10, n_{b r}=15\right)$.

A

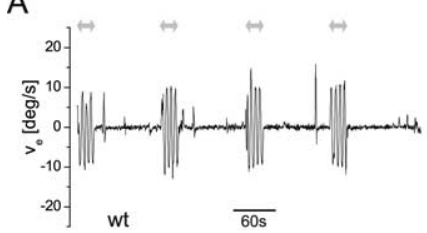

B

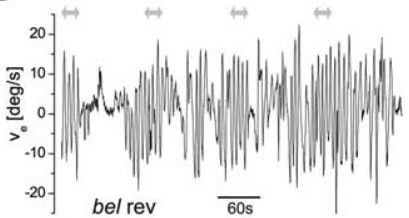

C

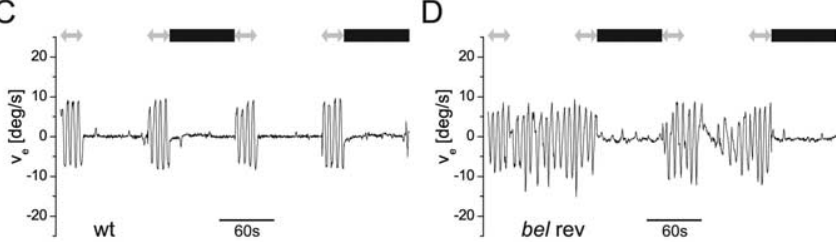

E

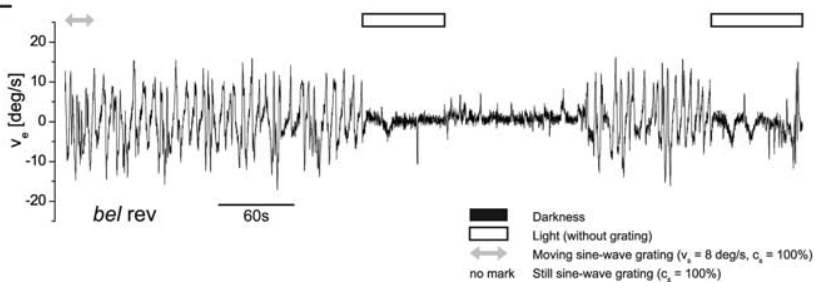

Figure 4. Eye velocity $\left(v_{e}\right)$ traces of wt and the spontaneous oscillations (SO) displayed by some bel rev. The $v_{e}$ traces were obtained from either a single wt or a single bel rev larva. $A$, Eye velocity of wt during moving and still grating. $\boldsymbol{B}$, Behavior of bel rev under identical conditions. Eye movements are observed without motion stimulus. $\boldsymbol{C}$, Eye velocity of wt when moving grating, still grating, and darkness were alternated. $\boldsymbol{D}$, bel rev under the same conditions. Oscillations are visual input dependent. $\boldsymbol{E}$, Eye velocity of bel rev in response to moving grating, still grating, and light exposure without grating. Sampling rate of the eye position recording were 5 samples/s $(\boldsymbol{A}-\boldsymbol{D})$ and 12 samples/s $(\boldsymbol{E})$. Eye velocity traces were smoothened around the spikes caused by saccades when the eye velocity exceeded $18 \%$. The traces were further smoothened with a three-frame running average. All gratings had a contrast of $100 \%$ and a spatial frequency of $0.055 \mathrm{c} / \mathrm{deg}$. Gray arrows indicate a moving grating at a stimulus velocity of $8 \% \mathrm{~s}$ with alternating direction every $3 \mathrm{~s}$. When a still grating was presented, no special mark was put. Black rectangles signify complete darkness, whereas white rectangles indicate mere light exposure without a grating.

lating when presented with a still grating (Fig. $4 A, B$ ). In complete darkness, the eyes of both wt and bel rev cease moving (Figs. $4 C, D)$. As a result, the oculomotor instability seen in bel rev cannot be attributed to a motor system instability (efferent) but must be caused by a sensory deficiency. Under illumination without grating, eye movements of bel rev discontinued (Fig. $4 E$ ). In addition, Figure $4 E$ also shows that the SOs occur at the sole pres- 


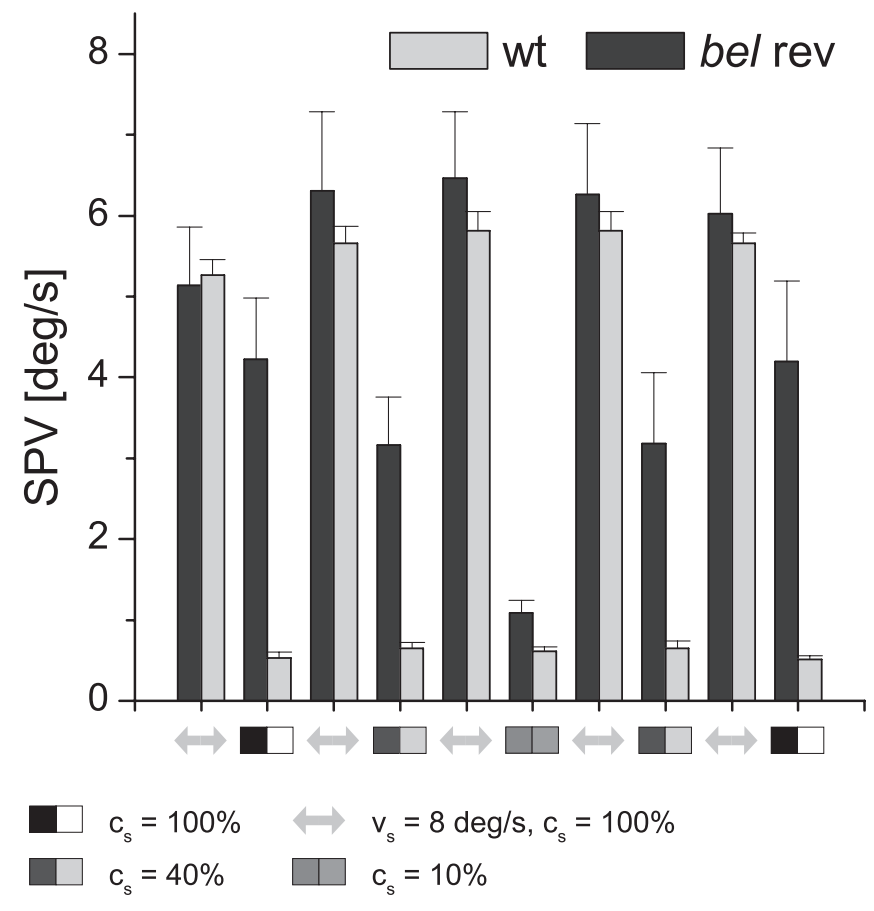

Figure 5. Stimulus contrast $\left(c_{s}\right)$ dependence of the spontaneous oscillations. Saccades were filtered when the eye velocity exceeded $18 \%$. The SPV was derived by calculating the mean of the absolute eye velocity per condition. Conditions with a contrast of $100 \%$ and a $v_{s}$ of $8 \%$ s were alternated with a still grating $\left(v_{s}=0 \% s\right)$ at differing contrasts $(100-40-10-40-100 \%)$. Light gray bars, wt $(n=8)$; dark gray bars, bel rev $(n=7)$.

ence of a still grating without previous triggering by a moving stimulus. Although the redisplay of the gratings did not, in most cases, reinitiate the SO immediately, they resumed shortly after, presumably subsequent to a saccade or another arbitrary neural trigger.

Because we have demonstrated a positive correlation between the reversed OKR and $c_{s}$, we wanted to elucidate the relationship between the SO and $c_{s}$. Thus far, we know that the eyes of bel rev oscillate at $100 \%$ contrast and remain stationary at $0 \%$ contrast. We presented gratings to bel rev and wt at varying $c_{s}$ in a moving and in a still version with each condition preceded by a moving grating. Figure 5 shows that the SOs are modulated by the $c_{s}$ similarly to the reversed OKR of bel rev and the normal OKR in wt larvae. Therefore, SOs are dependent on visual input but not on motion in the surround.

\section{Quantitative model}

To further prove that the reversed OKR and SOs are caused by the ipsilateral projection of the RGC in bel rev, we built a model to replicate the normal OKR with the transfer functions estimated based on bel fwd. We used bel fwd instead of wt because bel fwd, despite having a normally directed OKR, has a considerably reduced OKR gain (Fig. 3B). Therefore, besides the projection defect in bel rev, there may be other abnormalities present in bel larva that influence the OKR. Because, in bel rev, the RGCs project ipsilaterally but map to their correct tectal position, we expected the detected retinal slip velocity to be reversed. Accordingly, if we are able to make the model for the normal OKR produce a reversed OKR and SO simply by reversing the sign of the retinal slip velocity input to the OKR element, we provide additional credibility to the hypothesis that ipsilateral RGC projection is sufficient to produce reversed OKR and SO.

First, we calibrated the parameters of the OKR and the Ve
Load element in the model. The SPV versus mean retinal slip velocity curve was computed based on bel fwd data presented in Figure $3(n=18)$ and used for the parameter estimation of the OKR and Ve Load transfer function (Fig. 6B) (supplemental Table 1, available at www.jneurosci.org as supplemental material) $\left(R^{2}=0.9722\right)$. The resulting transfer function of the OKR element is presented in Figure 6C. The initial eye position, the initial eye velocity, and the saccade parameters such as the maximum eye range were estimated based on the corresponding empirical data set displayed in Figure 7, $A, C$, and $E$, because these parameters show remarkable between-subject variability. The normal OKR generated by the model is shown in Figure $7 \mathrm{~B}$. To replicate the projection defect in the OKR model, we changed the value of the gain element between the retinal slip velocity and the OKR element input to -1 . Through this modification, we were able to successfully replicate the reversed OKR (Fig. 7D) and the SO (Fig. $7 F$ ).

\section{Discussion}

bel rev display OKR and SO. Both of these behaviors are visualinput dependent and correlate with contrast. The unique SO closely resembles the involuntary eye movements in human patients affected with CN. bel encodes a Lim domain homeobox protein Lhx 2 , and the loss of its function specifically leads to disruptions in three major axon pathways in the forebrain, which are as follows: the anterior commissure, the post-optic commissure, and the optic chiasm at the midline (Seth et al., 2006). In bel homozygous mutants (bel fwd and rev), a variable fraction of RGC axons aberrantly project into the ipsilateral brain hemisphere that has been associated with a reversed OKR (Rick et al., 2000). To understand the causal chain between the optic misprojection and these abnormal oculomotor behaviors, we undertook an in-depth analysis of visual performance in achiasmatic bel larvae based on an optokinetic stimulation paradigm.

\section{Contrast sensitivity remains unchanged, and stimulus} velocity dependence is reduced in reversed OKR

The OKR has been used as a behavioral measure to examine the contrast sensitivity in both cats (Donaghy, 1980) and humans (Leguire et al., 1991; Harris and Smith, 2000). We have shown that the eye SPV in bel rev maintains a logarithmically linear function of stimulus contrast $\left(c_{s}\right)$ just as in wild-type larvae (Rinner et al., 2005) despite the sign reversal (Fig. 2A-D). Moreover, the similar slopes of the normalized SPV versus the logarithmical $c_{s}$ across the three phenotypes (wt, bel fwd, and bel rev) indicate that contrast sensitivity stays unaffected by the bel mutation (Fig. 2D-F). Statistical analysis of contrast sensitivity based on linear regression has confirmed this. Thus, the optokinetic performance of bel rev is influenced by visual input.

In the velocity challenge experiment, we observed differences in OKR performance among wt, bel fwd, and bel rev (Fig. 3). First of all, both wt and bel fwd reached their maximal SPV when the stimulus velocity was $32 \%$, whereas in bel rev, the SPV peak was reached much earlier at a stimulus velocity of $16 \%$ s. Second, the starting SPV $\left(v_{\mathrm{s}}=4 \%\right.$ s $)$ in bel rev was much higher than in the other two groups, considerably exceeding the stimulus velocity, hence leading to a slow-phase OKR gain $(\mathrm{SPG})>1$. Because bel rev and bel fwd carry the same mutation, the difference can be attributed to the achiasmatic condition of bel rev. Hence, the reversed sign of the retinal slip detection may account for the much higher SPG and the reduced stimulus velocity dependence at low stimulus velocities. In bel rev, the regenerative feedback 
loop of the OKR receives a sign-reversed error signal, causing the retinal slip to increase gradually.

At stimulus velocities beyond the SPV peak, all three groups showed a gradual decrease in SPV with increasing stimulus velocity. It is reasonable to explain the SPV and the SPG drop as a consequence of motion blurring caused by the failure of the OKR mechanism to compensate for the retinal slip, thus allowing for high retinal slip velocities to occur. Assuming that the sensory detection has a fixed-frame rate, motion blurring can be understood as a temporal integration of the stimulus in the retina, leading to reduced perceived $c_{s}$. Thus, the SPV decay can be explained in terms of a reduced perceived $c_{s}$. Because, in both bel fwd and bel rev, the maximum velocity reached during saccades is the same as in wt, the lower peak SPV in bel fwd and bel rev implies a sensory deficiency and/or a reduced efficiency of the OKR circuitry rather than a motor defect. At very high stimulus velocities, the eye stops following the moving grating as a consequence of motion blurring with the SPV converging to a theoretical asymptote at $\sim 1.5 \% \mathrm{~s}$ (noise floor of the SPV data). Finally, it is worth mentioning that, after both bel fwd and bel rev have reached their peak SPV, their curves essentially overlap (also the OKR gain curves), which well supports the idea that these two groups show a very similar OKR decay regardless of the reversed sign in bel rev. The weaker OKR of bel fwd and bel rev might be attributed to observed subtle retinal defects, particularly affecting amacrine cells of the retina (Seth et al., 2006).

Because we used a monocular stimulation paradigm in our OKR experiments, we were also interested in the behavior of the unstimulated eye, which may help us extend our knowledge about the cross talk between the left and right brain areas involved in the OKR. It turned out that, in bel rev, the unstimulated eye performed at a higher rate than the stimulated eye. In wt and bel fwd, on the other hand, the unstimulated eye showed a comparably weaker response. Interestingly, the multiplicative inverse of the left/right-eye gain in wt is comparable to the left/right-eye gain in bel rev. This may be because of a gain between the contralateral optic tectum and the ipsilateral motor neurons that increases the drive on the stimulated eye in wt. In bel rev, in contrast, that gain is missing in the stimulated eye but drives the unstimulated eye, leading to the reciprocal of the left/right-eye gain. This provides additional proof to the hypothesis that the optokinetic phenotype of bel rev is caused by the underlying RGC projection defect (data not shown).

\section{Both SOs and reversed OKR are caused by the achiasmatic projection}

The SOs have been only observed in bel rev. We have shown that this intriguing behavior requires visual input with a pattern presented and is contrast sensitive. All of these properties indicate that SOs have the same underlying cause as the reversed OKR.
B
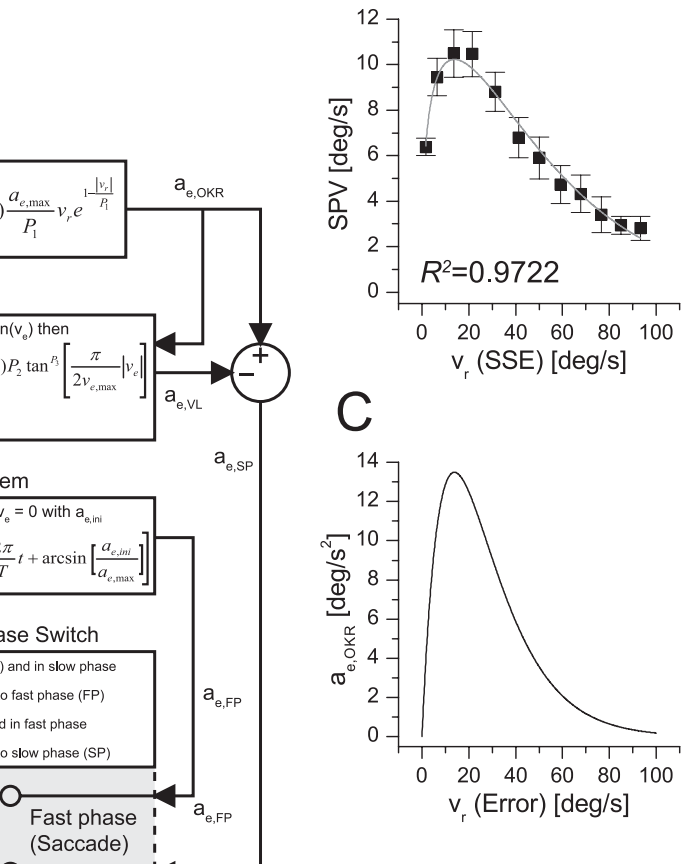

Slow phase; $a_{e, S P}$

$$
\text { (1) }
$$

Figure 6. A detailed model used for the computer simulation of the reversed OKR and the $S 0$ observed in bel rev. $A$, Diagram of

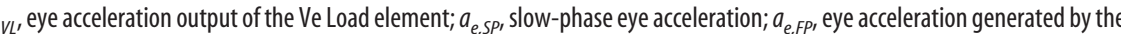
formulas used in the model blocks are discussed in supplemental Tables 1 and 2 and the supplemental movie (available at www.jneurosci.org as supplemental material).

Thus, the neural projection error in the brain is the main reason of both abnormal oculomotor behaviors.

The main function of the OKR is to stabilize the visual image on the retina. In this simple sensorimotor feedback system, the visual motion sensor detects the motion of the visual environment relative to the retina as an input signal that then elicits compensatory eye movements, the output response, to reduce the slip velocity of the retinal image. Thus, the retinal slip serves as the error signal in this closed feedback loop, and the eye adjust its velocity according to the perceived pattern velocity. The aim of this controlling negative feedback loop is to reduce the slip velocity ideally to zero. Based on this principle, we have built a quantitative model to simulate the neural circuit of OKR in larval zebrafish. In the achiasmatic condition (bel rev), the signal from the eye always feeds into the wrong hemisphere, leading to a nasal-temporal reversed perception. The attempt to compensate the retinal slip takes the wrong direction, thereby actually increasing the retinal slip. The resulting positive feedback loop is incapable of stabilizing the visual system, causing the observed, unstable oculomotor behaviors in bel rev. This idea is supported by the successful replication of the reversed OKR and the SO by reversing the sign of the error signal (retinal slip) in the model. Analogous to the model in which the initial eye velocity has to differ from zero, spontaneous eye movements such as a saccade are necessary to initiate $\mathrm{SOs}$ in bel rev. 


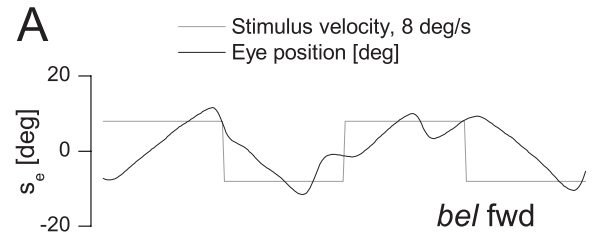

B

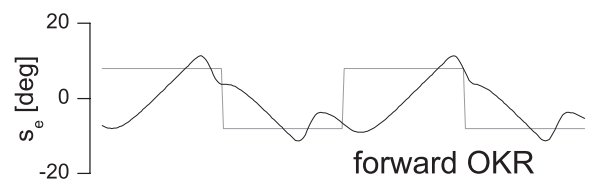

$\mathrm{C}$
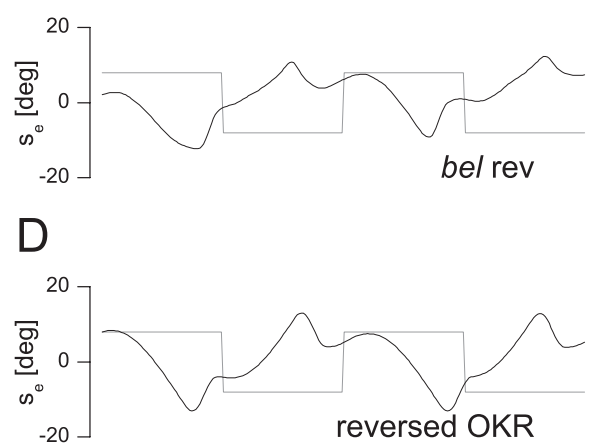

E

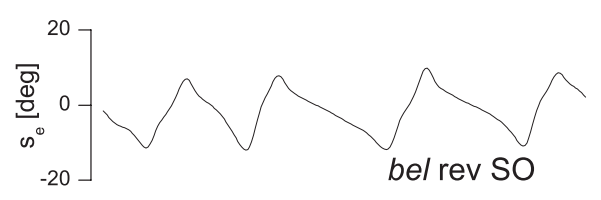

$\mathrm{F}$

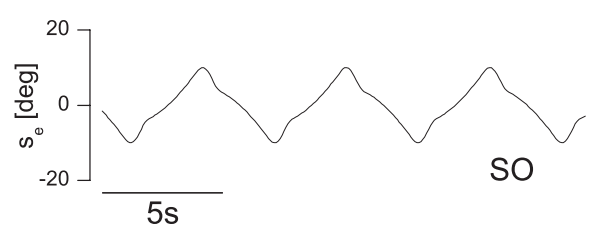

Figure 7. Comparison of the empirical eye position traces with model simulations. $A$, Eye position trace of a bel fwd larva. $\boldsymbol{B}$, Simulated normally directed OKR. C, Eye position trace of a bel rev larva. $\boldsymbol{D}$, Simulated reversed OKR. $\boldsymbol{E}$, Eye position trace of a bel rev larva exhibiting spontaneous oscillations. $\boldsymbol{F}$, Simulated spontaneous oscillations in which, in order for the model to start oscillating, it was necessary to set the initial eye velocity to a value differing from zero. Derived from the fish in $\boldsymbol{E}$, we used a value of -6.7 . Likewise, in bel larva, the oscillations are often initiated by saccades or small spontaneous eye resets. All model simulations were computed based on the parameters shown in supplemental Table 1 (available at www.jneurosci.org as supplemental material). We used a solver step size of 0.01 . The sample rate of the fish recording and the model readout was 12.5 samples/s.

\section{SOs closely resemble the $\mathrm{CN}$ in humans}

In belladonna, we have shown that the SO phenotype is most likely correlated with the underlying anatomical defect. Human patients affected with similar RGC projection defects also often suffer from an involuntary rhythmic eye movement, CN (Apkarian et al., 1994, 1995), which closely resembles the SO. It has been shown that a specific form of infantile nystagmus that is involuntary and conjugate, involving regular epochs of "active" and "quiet" phase (congenital periodic alternating nystagmus), is prevalent in $\mathrm{CN}$ associated with albinism (Abadi and Pascal, 1994). In some bel rev with a long recording time of SOs, we have observed analogous transitions in waveform characteristics. Our model is capable of replicating these waveforms and even a sudden stop of the SO: equal signs of the pre- and post-saccadic $\left(v_{e, \text { exit }}\right)$ eye velocity produce a beating nystagmus (jerk nystagmus), whereas different signs cause a pendular nystagmus. Random variation of the post-saccadic eye velocity leads to a periodic alternating nystagmus. Finally, the SOs cease when the postsaccadic eye velocity equals zero (data not shown).

Another abnormity we have observed in bel rev is frequently occurring spinning swimming behavior. This moving instability could well be related to failure of the eye to lock on an object in the visual field. In patients with vestibular neuritis, for instance, voluntary suppression of nystagmus reduces postural sway and increases balance (Jahn et al., 2002; Glasauer et al., 2005). Also, here nystagmus is associated with postural instability, further supporting the link between $\mathrm{CN}$ in humans and the SO in bel rev.

In contrast to achiasmatic mammals with binocular vision, bel rev provides a relatively clean model for studying visual behaviors in relation to optic nerve projection defects. The conflict between correct and erroneous information in binocularity is one explanation why, in patients with NDRFF and albinism, the OKR profile is complex, and reversed OKR has not been observed. In NDRFF syndrome patients, misprojections are mixed with remaining correct ones, and as also the case in healthy humans, some RGCs project ispilaterally. Similarly, in albinism patients, about half of the axons correctly project to the contralateral hemisphere, whereas those destined for the ipsilateral hemisphere project contralaterally as well. Because fish have monocular vision, all of the optic fibers cross the midline forming the optic chiasm and project to the contralateral hemisphere. The half brain of the achiasmatic fish therefore receives only visual information from the "wrong" eye. This gives us a unique chance to study the visual defect caused by retinotectal axon pathfinding errors directly.

We have linked the reversed OKR and CN to the same underlying neural circuit defect, using the mutant belladonna as a behavioral tool. In this study, we used the mutant as a behavioral model of oculomotor instabilities such as nystagmus. The underlying molecular nature of the mutation is not informative in this context. Indeed, we drew comparison to the human condition that is, in all likelihood, not molecularly but behaviorally comparable to bel.

\section{References}

Abadi RV, Pascal E (1994) Periodic alternating nystagmus in humans with albinism. Invest Ophthalmol Vis Sci 35:4080-4086.

Apkarian P, Bour LJ (2001) See-saw nystagmus and congenital nystagmus identified in the non-decussating retinal-fugal fiber syndrome. Strabismus 9:143-163.

Apkarian P, Bour L, Barth PG (1994) A unique achiasmatic anomaly detected in non-albinos with misrouted retinal-fugal projections. Eur J Neurosci 6:501-507.

Apkarian P, Bour LJ, Barth PG, Wenniger-Prick L, Verbeeten Jr B (1995) Non-decussating retinal-fugal fibre syndrome. An inborn achiasmatic malformation associated with visuotopic misrouting, visual evoked potential ipsilateral asymmetry and nystagmus. Brain 118:1195-1216.

Collewijn H, Grootendorst AF (1978) Adaptation of the rabbit's vestibuloocular reflex to modified visual input: importance of stimulus conditions. Arch Ital Biol 116:273-280.

Collewijn H, Apkarian P, Spekreijse H (1985) The oculomotor behaviour of human albinos. Brain 108:1-28.

Dell'Osso LF, Williams RW (1995) Ocular motor abnormalities in achiasmatic mutant Belgian sheepdogs: unyoked eye movements in a mammal. Vision Res 35:109-116.

Dell'Osso LF, Williams RW, Jacobs JB, Erchul DM (1998) The congenital 
and see-saw nystagmus in the prototypical achiasma of canines: comparison to the human achiasmatic prototype. Vision Res 38:1629-1641.

Donaghy M (1980) The contrast sensitivity, spatial resolution and velocity tuning of the cat's optokinetic reflex. J Physiol (Lond) 300:353-365.

Easter Jr SS, Schmidt JT (1977) Reversed visuomotor behavior mediated by induced ipsilateral retinal projections in goldfish. J Neurophysiol 40:1245-1254.

Feinsod M (2000) The blind beautiful eye. J Neuroophthalmol 20:22-24.

Glasauer S, Schneider E, Jahn K, Strupp M, Brandt T (2005) How the eyes move the body. Neurology 65:1291-1293.

Haffter P, Granato M, Brand M, Mullins MC, Hammerschmidt M, Kane DA, Odenthal J, van Eeden FJ, Jiang YJ, Heisenberg CP, Kelsh RN, FurutaniSeiki M, Vogelsang E, Beuchle D, Schach U, Fabian C, Nusslein-Volhard C (1996) The identification of genes with unique and essential functions in the development of the zebrafish, Danio rerio. Development 123:1-36.

Halmagyi GM, Gresty MA, Leech J (1980) Reversed optokinetic nystagmus (OKN): mechanism and clinical significance. Ann Neurol 7:429-435.

Harris LR, Smith AT (2000) Interactions between first- and second-order motion revealed by optokinetic nystagmus. Exp Brain Res 130:67-72.

Hogan D, Williams RW (1995) Analysis of the retinas and optic nerves of achiasmatic Belgian sheepdogs. J Comp Neurol 352:367-380.

Jahn K, Strupp M, Krafczyk S, Schuler O, Glasauer S, Brandt T (2002) Suppression of eye movements improves balance. Brain 125:2005-2011.

Jeffery G (1997) The albino retina: an abnormality that provides insight into normal retinal development. Trends Neurosci 20:165-169.

Karlstrom RO, Trowe T, Klostermann S, Baier H, Brand M, Crawford AD, Grunewald B, Haffter P, Hoffmann H, Meyer SU, Muller BK, Richter S, van Eeden FJ, Nusslein-Volhard C, Bonhoeffer F (1996) Zebrafish mutations affecting retinotectal axon pathfinding. Development 123:427-438.
Korff CM, Apkarian P, Bour LJ, Meuli R, Verrey JD, Roulet Perez E (2003) Isolated absence of optic chiasm revealed by congenital nystagmus, MRI and VEPs. Neuropediatrics 34:219-223.

Leguire LE, Zaff BS, Freeman S, Rogers GL, Bremer DL, Wali N (1991) Contrast sensitivity of optokinetic nystagmus. Vision Res 31:89-97.

Mullins MC, Hammerschmidt M, Haffter P, Nusslein-Volhard C (1994) Large-scale mutagenesis in the zebrafish: in search of genes controlling development in a vertebrate. Curr Biol 4:189-202.

Neuhauss SC, Biehlmaier O, Seeliger MW, Das T, Kohler K, Harris WA, Baier H (1999) Genetic disorders of vision revealed by a behavioral screen of 400 essential loci in zebrafish. J Neurosci 19:8603-8615.

Rick JM, Horschke I, Neuhauss SC (2000) Optokinetic behavior is reversed in achiasmatic mutant zebrafish larvae. Curr Biol 10:595-598.

Rinner O, Rick JM, Neuhauss SC (2005) Contrast sensitivity, spatial and temporal tuning of the larval zebrafish optokinetic response. Invest Ophthalmol Vis Sci 46:137-142.

Seth A, Culverwell J, Walkowicz M, Toro S, Rick JM, Neuhauss SC, Varga ZM, Karlstrom RO (2006) Belladonna/(lhx2) is required for neural patterning and midline axon guidance in the zebrafish forebrain. Development 133:725-735.

Shallo-Hoffmann J, Faldon M, Tusa RJ (1999) The incidence and waveform characteristics of periodic alternating nystagmus in congenital nystagmus. Invest Ophthalmol Vis Sci 40:2546-2553.

St John R, Fisk JD, Timney B, Goodale MA (1984) Eye movements of human albinos. Am J Optom Physiol Opt 61:377-385.

Wilhelm H, Wilhelm B, Schiefer U (1991) Mydriasis caused by plant contact. Fortschr Ophthalmol 88:588-591.

Williams RW, Hogan D, Garraghty PE (1994) Target recognition and visual maps in the thalamus of achiasmatic dogs. Nature 367:637-639. 